\title{
Assembly Concept and Technology of the ATLAS Barrel Toroid
}

\author{
A. Foussat, H. H. J. ten Kate, B. Levesy, C. Mayri, Y. Pabot, V. Petrov, M. Raymond, Z. Sun, and P. Vedrine
}

\begin{abstract}
The ATLAS detector for the LHC collider at CERN requires a large superconducting Barrel Toroid (BT) with overall dimensions of $25 \mathrm{~m}$ length, $22 \mathrm{~m}$ diameter which is installed in the ATLAS cavern 100m underground. The Barrel Toroid provides the magnetic field for the muon detector. The toroid is assembled from 8 flat race track coils of dimensions $25 \mathrm{~m} \times 5 \mathrm{~m}$. Following the on-surface acceptance test of the 8 BT coils, they are successively inserted in the underground cavern and assembled as a full toroid by using 16 supporting rings of struts that link the 8 coils to form a rigid and stable structure. The total mass of the toroid is $850 \mathrm{t}$. Particular issues are that the axis of the toroid has to be horizontal and the final shape of the toroid cylindrical with a tolerance of $+/-10$ $\mathrm{mm}$ with respect to an overall system diameter of $22 \mathrm{~m}$. The desired shape of the toroid can only be controlled by installing the 8 coils in calculated positions in space (forming an elliptic shaped structure), and by linking them using bolted struts linking the coils. The final release of the structure is done under its self weight hydraulically. This required very large tooling essentially to support all the 8 coils in space in a nearly stress free condition until the toroid supporting rings have been closed. The theoretical positions are found by performing detailed 3-D Finite Element Calculations that predict the shape of the toroid under its operational load. The assembly of this huge toroid is unique and no experience basis exists.

This paper presents the concept and technology required for the assembly of the toroid as well as the cryogenic supply lines, highlights the FEA mechanical calculations performed to predict the shape, summarizes the tooling required and reviews the experience gained during the installation.
\end{abstract}

Index Terms-ATLAS detector, cryogenics, mechanical installation, superconducting magnet, toroid.

\section{INTRODUCTION}

$\mathbf{T}$ THE ATLAS Barrel Toroid (BT) is a large air-core magnet that provides the magnetic field needed for the ATLAS muon spectrometer operation. The ATLAS Barrel Toroid has been designed by CEA-Saclay in collaboration with the ATLAS Magnet group at CERN. The Barrel Toroid structure, named Warm Structure, holds the eight racetrack coils sized $(25 \mathrm{~m} \times$ $5 \mathrm{~m}$ ) (Fig. 1) evenly positioned around the proton-proton beam axis. The outer diameter of the structure is $22 \mathrm{~m}$ [1]. The warm structure supports not only the coils but also muon detectors, services and access structures for the ATLAS experiment, which brings the total mass to $1400 \mathrm{t}$. When energizing the toroid, the warm structure is reacting the integral centering magnetic forces

Manuscript received September 18, 2005.

A. Foussat, H. H. J. ten Kate, and V. Petrov are with the European Organization of Nuclear Research (CERN), 1211 Geneva 23, Switzerland (e-mail: arnaud.foussat@cern.ch).

M. Raymond is with CPPM/IN2P3, Marseille 13009, France.

B. Levesy, C. Mayri, Y. Pabot, Z. Sun, and P. Vedrine are with CEA DSM/ DAPNIA, 91191 Gif-sur-Yvette Cedex, France.

Digital Object Identifier 10.1109/TASC.2005.864352

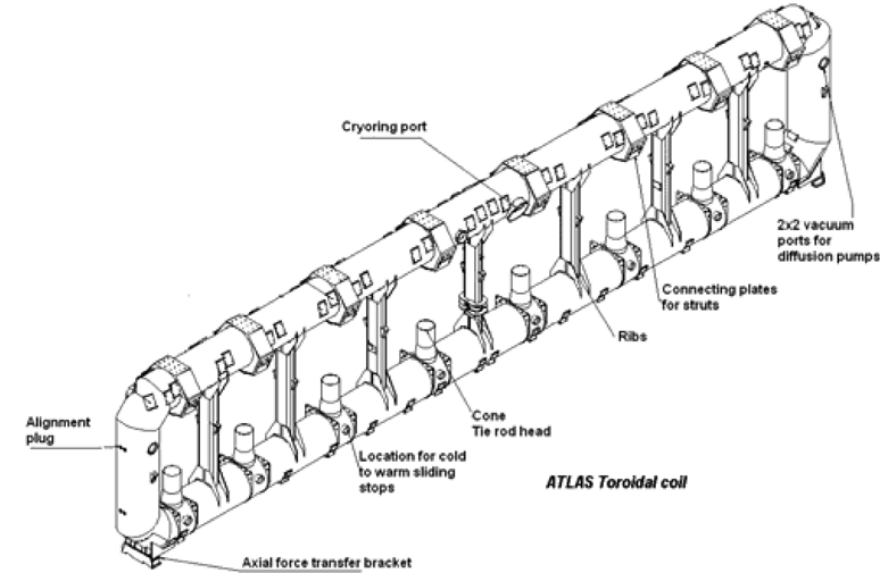

Fig. 1. Isometric view of single BT coil.

of maximal 1.3 MN per coil. Due to the size of the BT coils, it was chosen to test each coil on surface and to perform the global commissioning test in the ATLAS experimental cavern once the mechanical installation is complete and services are connected.

The design of the BT mechanical structure required detailed finite element analyses to determine deformations and stress levels in the magnet structure. The consequences resulting from the calculations were many: it influenced directly the assembly strategy like the nominal shape of installation, the technology used in the bolting interfaces and the mechanical specifications of the warm structure components. The detailed design was further influenced by the available manufacturing techniques for the rather unique structural elements. The BT installation required various technical services support such as extensive use of metrology, thorough quality control in particular on bolting, mechanical workshop for machining the shims, cryogenic transfer lines and the highest level of operational safety on site. For various heavy lifting and to keep the coils in position during assembly special heavy handling tools and support structures were required.

\section{BASIC CONCEPT}

\section{A. Scope of Installation}

The Barrel Toroid comprises 16 sectors, alternatively including a BT coil and Warm Structure of aluminum beams called struts (outer ring) and voussoirs (inner ring) (Fig. 2). The warm structure provides the support and structural stabilization of the coils in the Barrel Toroid. Each coil and components are transferred to the cavern sequentially according to the installation plan which minimizes deformations, and the associated technical risk. Each following sector is assembled 


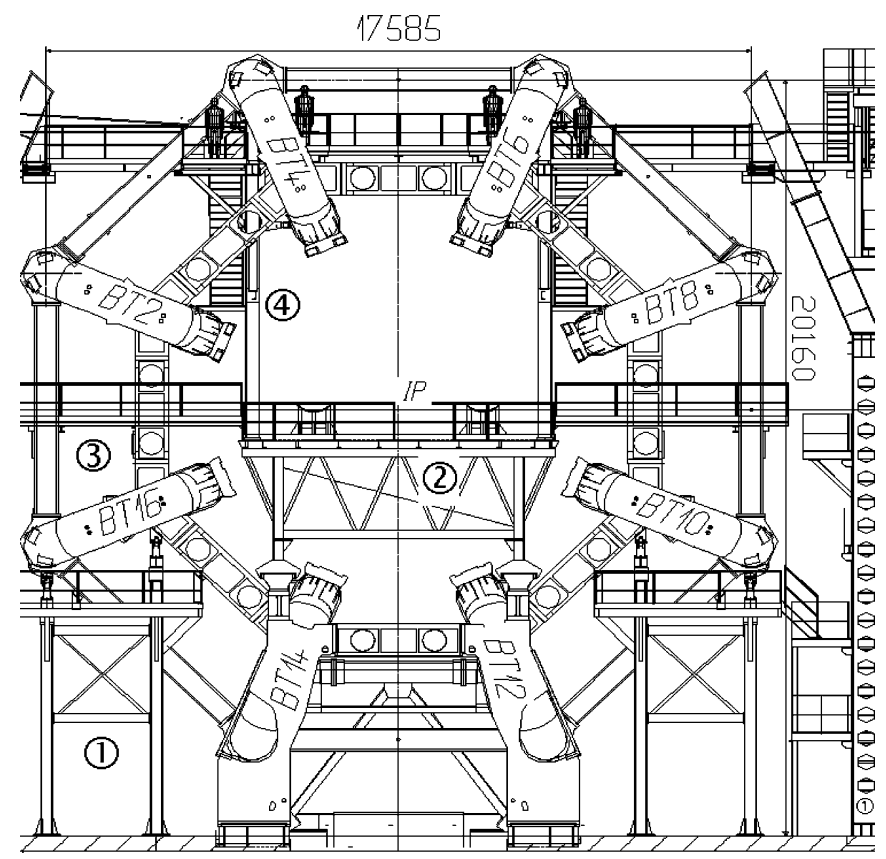

Fig. 2. Cross sectional view of Barrel Toroid coils and warm structure with temporary associated installation tooling: (1) lateral hydraulic supports, (2) central platform on rails, (3) extension access platforms, (4) top hydraulic columns supports.

in such a way that the next coils are installed in a nearly stress-free condition using large temporary metallic supports. To compensate for the sagging of the toroid under self weight and extra detector service loads, the coils are positioned on an ellipse with eccentricity parameters vertical $\Delta y=+29 \mathrm{~mm}$, horizontal $\Delta x=-15 \mathrm{~mm}$ [2]. After releasing the temporary supports by hydraulic jacks, the final circular shape is expected. The barrel (Fig. 2) is constructed symmetrically around the beamline starting with the installation of the first two bottom coils resting on the ATLAS feet bearing in total $7000 \mathrm{t}$ of the experiment.

The specified tolerance of positioning individual coils is $+/-5 \mathrm{~mm}$ in all directions and the assembled 8 coils should be finally positioned on a circle with an accuracy of $+/-10$ $\mathrm{mm}$. Considering the alignment accuracy close to the limit of what is achievable, the accumulation of deviations must be strictly controlled and each geometrical interface needs to have provision for adjustment included in the design. This is achieved by means of 3-D aluminum alloy Al 5083 shims $\left(500 \times 850 \times 15 \mathrm{~mm}^{3}\right)$ machined on demand to $0.1 \mathrm{~mm}$ accuracy and inserted in all connections between coils and warm structure. A detailed dimensional survey at completion of each sector provides the geometrical estimate of the magnetic datum for the as-built barrel toroid and the reference for the following warm structure sectors.

\section{DESIGN AND MANUFACTURING}

\section{A. Mechanical Structure Calculations}

A global finite element model of the Barrel Toroid (205000 brick elements) has been developed at CEA-Saclay and CERN

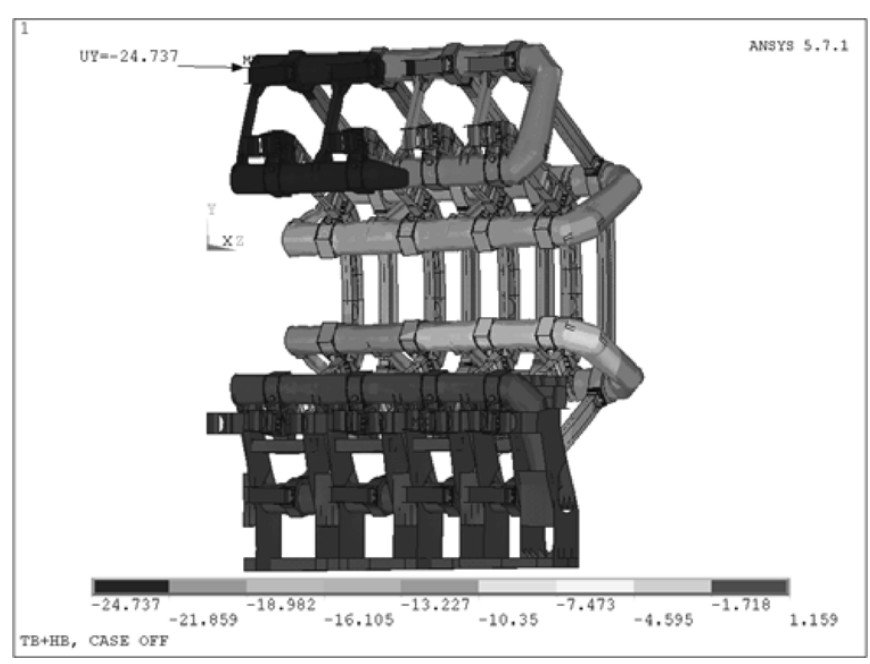

Fig. 3. Global BT warm structure FEA model used to derive resultant forces on bolted interfaces, deformation, and stress levels.

TABLE I

MATERIAL PROPERTIES

\begin{tabular}{|c|c|c|}
\hline Items - features & Material & $\begin{array}{c}\text { Yield stress } \\
\mathrm{Rp}_{0.2} / \text { Tensile } \\
\text { stress }(\mathrm{MPa}) \\
\end{array}$ \\
\hline $\begin{array}{l}\text { Studs M42/M36 x } \\
290-325 \mathrm{~mm}\end{array}$ & $\begin{array}{l}\text { X6NiCrTIMoVB 25.15.2 } \\
\text { (Grade I), Beck Crespel } \\
\text { Company (Fr) }\end{array}$ & $620 / 900$ \\
\hline Studs M42/M36 x & A2-50 (Grade II $)$ & $210 / 500$ \\
\hline $290-325 \mathrm{~mm}$ & A2-60 (Grade III) & $\begin{array}{c}345 / 620 \\
Z=15 \%\end{array}$ \\
\hline $\begin{array}{l}\text { Voussoirs/ Struts } \\
\text { beams }(2.85 \mathrm{~m} / 5.7 \mathrm{~m})\end{array}$ & Alu alloy Al5083 H111 & $175 / 255$ \\
\hline Vacuum vessel & Stainless steel SS304L & 175 \\
\hline $\begin{array}{l}\text { Aluminum shims } \\
500 \times 600 \mathrm{~mm}\end{array}$ & Alu alloy Al5083 H111 & $175 / 255$ \\
\hline $\begin{array}{l}\text { Aluminum Connection } \\
\text { boxes }\end{array}$ & $\begin{array}{l}\text { Alu alloy ( } \mathrm{Al} \mathrm{Zn} 4 \mathrm{Mg} 2 \mathrm{Mn} \text {, } \\
\text { Russian standard 1980T1 }\end{array}$ & $<230$ \\
\hline
\end{tabular}

(Fig. 3) to extract the deformations and resultant forces, which transit through the warm structure components and connections [2]. The boundary conditions included gravity, external atmospheric pressure, muon services load, radial magnetic forces as well as axial magnetic forces applied on both ends. A local model of one warm structure ring was then developed using contact elements with models of studs to dimension the bolts.

The maximum tensile force of $250 \mathrm{kN}$ per bolt (M36) was found in the inner voussoirs ring and a shear force per bolt (M42) of $20 \mathrm{kN}$ on the outer struts ring. According to the resultant calculated tensile and shear forces in each interface, the material of stud was selected among 3 grades of austenitic stainless steel (Table I). In order to keep good contact and to withstand shear forces by friction at the interfaces, pre-stressed bolting was chosen in combination with sand blasted shim surfaces to reach $\mu>0.4$ friction coefficient. The design value of the prestress per bolt was defined according to Eurocode 3 at $80 \%$ of the yield stress value. An empirical strain relaxation of $15-20 \%$ due to redistribution of mechanical play was measured. In order to reach the design tightening value, it is necessary to pre-stress to a higher value of $95 \%$ of yield stress. 

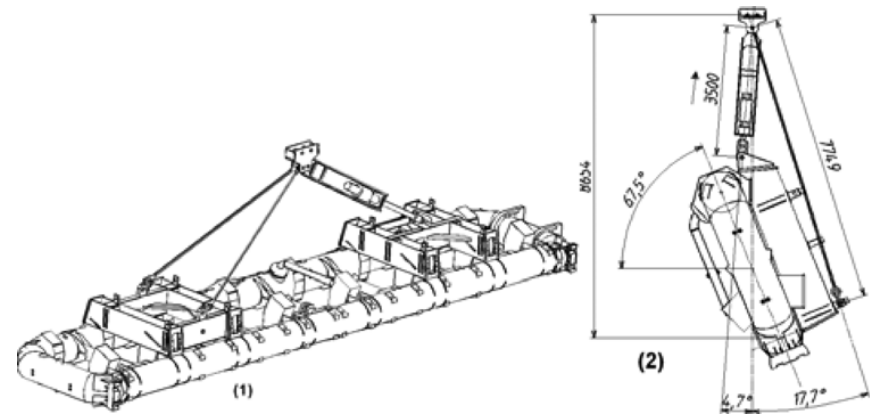

Fig. 4. Lifting tooling based on cable strand jacks used for descent of BT coil into experimental cavern (1) and angular positioning (2).

\section{B. Tooling}

1) Transport, Craning and Tilting Tools: Considering the large dimensions of the individual stainless steel 304L vacuum vessels, it is essential to limit stress and deformation to preserve the internal cold mass suspension system during any handling phase. The maximum deformation criterion is set to $1.7 \mathrm{~mm} / \mathrm{m}$ on the vacuum vessel which corresponds to a shear stress of 10 $\mathrm{MPa}$ at the conductor-bond interface and results in admissible sag of $21 \mathrm{~mm}$ across the coil length. All supports of the vacuum vessel are designed to keep the contact stress under the admissible buckling limit of $120 \mathrm{MPa}$. In order to meet the deformation and acceleration requirements $\left(\delta<2 \mathrm{~mm} / \mathrm{m}, \mathrm{a}_{\mathrm{i}}<0.3 \mathrm{~g}\right)$ during transport, a special trailer is used to carry the $120 \mathrm{t}$ coil. It is equipped with 6 hydraulic axles, to maintain an isostatic load configuration and to compensate for the road slope up to $4 \%$. Once the coil is unloaded in the surface hall, it is equipped with dedicated lifting tooling required to descend the coil in the experimental cavern through the $18 \mathrm{~m}$ diameter shaft. This tooling is composed of two main frames (see Fig. 4) bolted to the vacuum vessel and an industrial $150 \mathrm{t}$ capacity hydraulic cable strand jack. This jack allows to increase on demand the effective length of the sling with $\mathrm{mm}$ resolution in order to incline the coil during the descent in the shaft up to $60^{\circ}$ from horizontal.

During the lift on surface with the $140 \mathrm{t}$ capacity overhead crane, a maximum sag of $20 \mathrm{~mm}$ along the coil was measured.

2) Dedicated Mechanical Supports: The temporary mechanical supports used for the BT installation were designed by the magnet integration team at CERN according to Eurocode 3 standard and manufactured at the JINR nuclear research institute (Dubna, Russia). The main purpose of the structures is to provide hydraulic support interfaces to the BT and also serve as access platform at various heights. The installation scheme (Fig. 2) shows the bottom lateral supports with 4 groups of 8 jack cylinders with $93 \mathrm{t}$ capacity which support the 4 center coils. In the central region of the Barrel Toroid are located platforms used to receive coils from the surface. The central platform is equipped with columnar structures for supporting the top two coils as shown in Fig. 5. Once the last top sector is closed, the toroid structure is released gradually under its self load using sets of hydraulic jacks while respecting the deformation criterion on the vacuum vessel $(<2 \mathrm{~mm} / \mathrm{m})$. During this phase, a vertical displacement of $-29 \mathrm{~mm}$ is expected on the top sector and a maximum outwards lateral movement of $15 \mathrm{~mm}$.

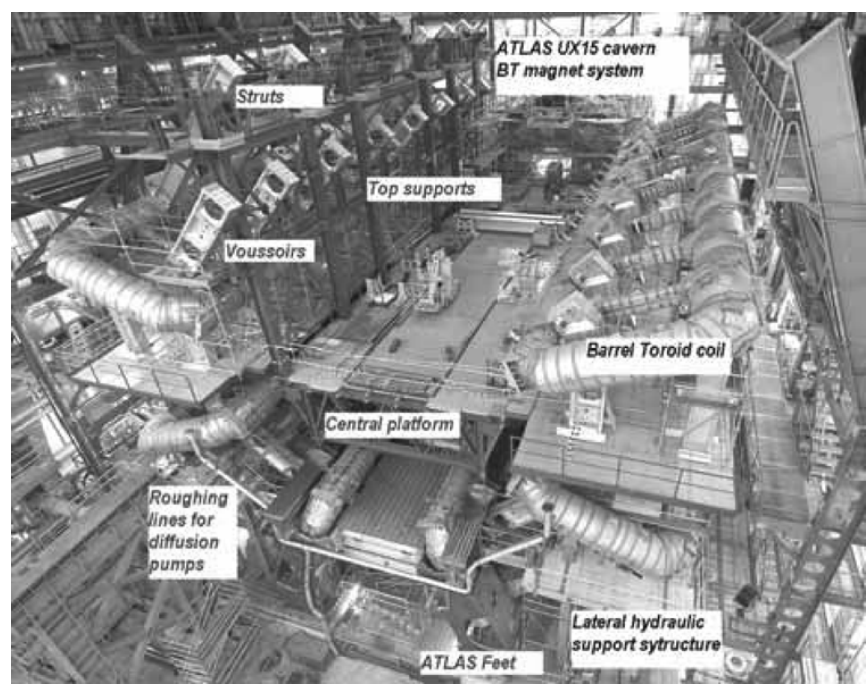

Fig. 5. Overall picture of ATLAS Barrel Toroid magnet system.

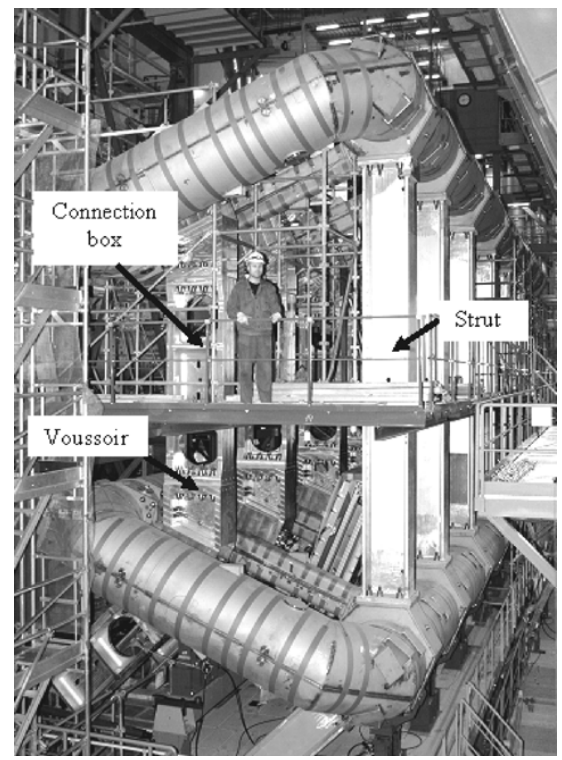

Fig. 6. Sector of warm structure on Barrel Toroid.

\section{Warm Structure Components}

The toroid is composed of 8 stainless steel vacuum vessels, as well as 8 inner and outer structural rings of aluminum beams named respectively voussoirs and struts. The mechanical features of each component are reported in Table I. Eight aluminum connection boxes of $1.3 \mathrm{t}$ each are pre-mounted on the coil tie rods heads to provide interface with voussoirs (Fig. 6). Those aluminum components are realized by stamping and extrusion at Samara Metallurgic plant (Russia). The design and manufacture of the Atlas Barrel Toroid Warm Structure components is described in [3].

\section{Warm Structure Interfaces Bolting}

The elements of the warm structure are linked using high strength bolting to withstand the large forces passing through 


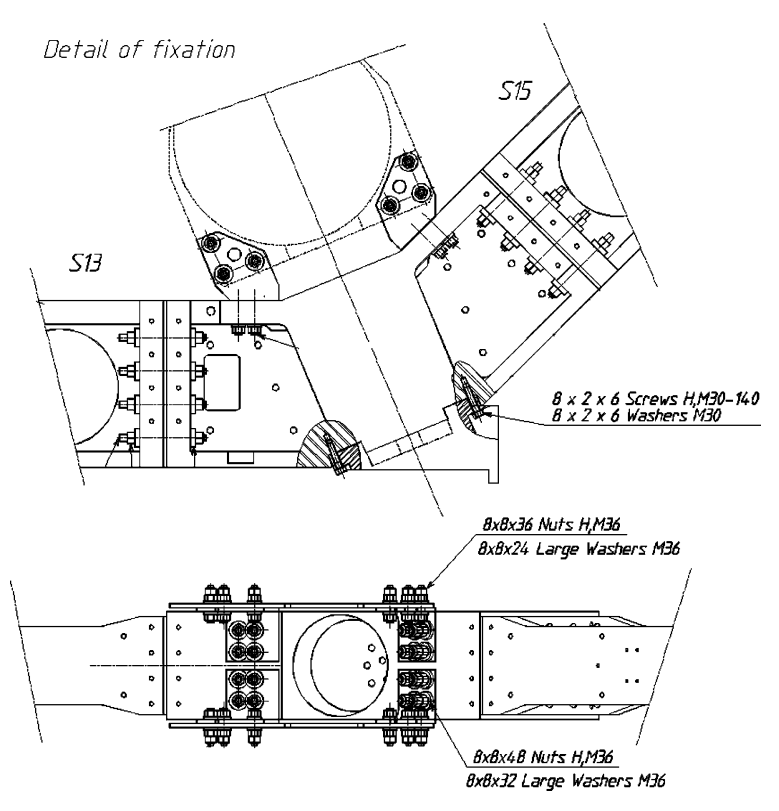

Fig. 7. Detail of high strength bolting on Voussoir-Connection box.

the connections and to built a rigid structure, a total of 5800 fasteners with specified relative magnetic permeability $\mu_{r}$ less than 1.2 .

Connection Boxes-Tie Rod Head: The maximum tensile magnetic force expected on a tie rod head is $2100 \mathrm{kN}$. Therefore, $18 \mathrm{M} 30$ rods are pre-stressed at $150 \mathrm{kN}$ to fix the connection boxes to the tie rod head.

Coil Connection to Voussoirs and Struts Elements: The tensile and shear forces applied on the warm structure bolted interfaces are function of the sector position on the toroid. A total of 6 studs M42 are used to connect the vacuum vessel to the struts and 16 studs M36 between the boxes to the Voussoirs (Fig. 7). A pre-stress of $500 \mathrm{kN}$ is applied on the high strength studs of the voussoir interfaces and $360 \mathrm{kN}$ on the struts connections.

\section{ASSEMBly}

\section{A. Organization}

The management of the toroid assembly was performed by the ATLAS magnet integration team which has the overall responsibility for cost of construction, to meet the installation milestones and assure technical control at industrial suppliers' premises. The installation project manager had to define the assembly procedures, to analyze and decide upon design changes or deviations. The manpower resources were allocated on work packages and covered by a few specialized teams from the High Energy Physics institutes (JINR, IHEP Russia). The overall manpower requirement was estimated at 26000 men hours for the mechanical installation excluding the services integration tasks. The quality insurance was supported by a team from CEA to implement controls on interfaces bolting, cryogenics pipes assembly (leak test, pressure test, $\mathrm{X}$ rays checks) and to maintain installation follow up databases.

\section{B. Outline Assembly Procedure}

The tight installation tolerances required on the assembled warm structure impose to follow sequences and processes which minimize both deviations and residual stresses so that the alignment errors do not accumulate. The assembly plan is designed to correct alignment deviations at each step of BT installation by using 3D aluminum shims at every bolted interface. Once the warm structure aluminum beam elements are pre-assembled in a given sector, the dedicated support tooling is properly aligned and the forthcoming coil can then be positioned.

1) Magnet Positioning Method: Each installation of a coil is preceded by a comprehensive survey of its geometry and interfaces which is used to define the "as-built BT coil datum". This constitutes the operational coil reference that includes manufacture deviations and will be targeted as installation shape within the references in ATLAS experimental cavern. During the assembly of a warm structure sector, a theodolite is used to perform accurate measurement of the linking beams positions and to implement adjustments to connect as-built matching coils. Similarly, the dedicated support tooling is adjusted to installation references including deviations from nominal up to $6 \mathrm{~mm}$. Each BT coil is then rigged with two underground $65 \mathrm{t}$ cranes connected to heavy handling tooling, tilted to azimuthal angle $\left(+/-22.5^{\circ},+/-67.5^{\circ}\right)$ using 2 hydraulic cable strand jacks (see Fig. 4) and positioned on provisional supports. At this stage, a survey of the coil interface planes is performed to locate the coil in the cavern space and to define its deviations from the so-called "elliptical BT installation datum". The final corrective movements on each interface for voussoirs and struts are applied using hydraulic groups of coupled jack cylinders. The installation position is then blocked on safety locking nuts or jacking screws depending on sectors. For each of the 16 interface points between the vacuum vessel and the warm structure, the gaps are measured accurately and corresponding 3D shims are machined with $0.1 \mathrm{~mm}$ accuracy and sand blasted. The mean thickness of the machined shims is $16 \mathrm{~mm}$. Once the shims are produced and inserted to fill the gaps using local jacking system, the interface is bolted with hydraulic tensioners actuated by a hydro-pneumatic pump.

The M36 and M42 studs are fitted with spherical bearing and highly preloaded up to $90 \%$ of the yield stress to ensure a good rigidity of the assembly joint. Each coil is linked to the warm structure by 352 bolts. A statistical control of the tightening is performed on about $12 \%$ of the bolts by ultrasonic method that measures the extension of the stud. A final survey of main fiducial marks on the vacuum vessel shows that the accuracy of positioning of a coil is typically $2.5 \mathrm{~mm}$ in radial direction and better than $1 \mathrm{~mm}$ along the toroid axis.

2) Installation of Cryogenic Transfer Lines: The purpose of the ring of cryogenic transfer lines is to house the electrical 22 kA bus-bars and to convey helium coolant to the cold masses and the thermal shields. The current is provided via aluminum stabilized $\mathrm{NbTi} / \mathrm{Cu} \mathrm{SC}$ bus bars cooled by supercritical helium at $4.7 \mathrm{~K}$. The helium at $4.7 \mathrm{~K}$ and $60 \mathrm{~K}$ is supplied by pipes with a pressure between 1.4 and 18 bar. The items are protected by a super-insulated thermal shield inside a vacuum vessel. The cryoring is divided in 8 sectors, located between the coils (Fig. 8) at 


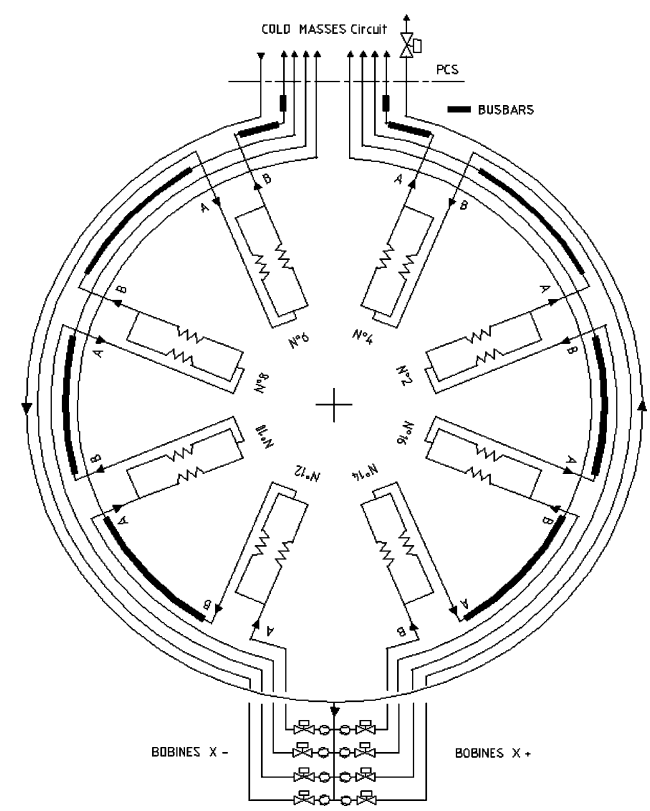

Fig. 8. Schematics of cryogenics LHe circuit connections inside cryoring sections between BT coils and bottom distribution valve box.

the external radius each sector of 6 meters long is mechanically installed as coil sectors are assembled and the interconnections are performed and tested.

The top section is linked to the Current Leads Cryostat which includes the supply lines for helium provided by the Proximity Cryogenic System. The bottom one includes a valve box allowing the fluid distribution to the eight coils. Each sector extremity is equipped with a sleeve allowing the connection to the coil during the toroid assembly. After alignment of the sector according to the coil cryoports with its sleeves opened, it is fixed to the closest aluminum strut. The first task in the sleeve area is to realize the electrical joint by TIG welding (Fig. 9) between the conductors in hand-shake configuration coming from the coil and those from the sector. Then the conductors are insulated and fixed using fiberglass impregnated with Damival epoxy resin and housed in a stiffening aluminum cooling casing.

The electrical bus-bar insulation level is then checked at $1 \mathrm{kV} \mathrm{DC}$. The design of the He cryogenic circuit allows a similar configuration of piping in each sleeve.

The cryogenic connections are adjusted, and welded in the sleeve with full X-ray radiography control in situ. The tightness of the whole piping is checked under 30 bar of helium gas

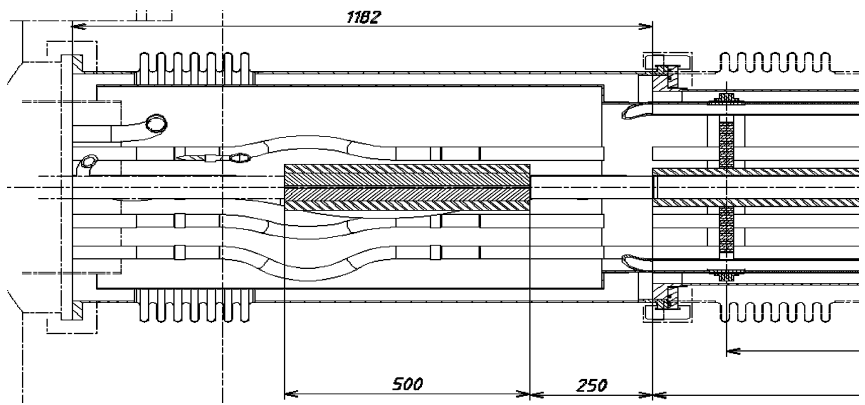

Fig. 9. Cross section of one end sleeve of cryogenics ring section, view of SC bus-bars junction.

and pumped up for leak tightness cross check with acceptance criteria of 10-8 mbar $1 \mathrm{~s}^{-1}$. Some lines are then instrumented with PT100 type temperature sensors. Each line is wrapped with super-insulation. The thermal shield, in two parts, is placed and wrapped with two super-insulation blankets. To achieve the tightness of the common vacuum vessel, the sleeve is closed on the cryoport flange of the coil.

\section{SUMMARY}

The ATLAS barrel toroid is under construction in the experimental cavern at the LHC accelerator. The mechanical installation of the toroid is complete and the magnet services are now being installed for the final cryogenic and power commissioning test.

\section{ACKNOWLEDGMENT}

The authors would like to thank the contributors to the ATLAS Barrel Toroid installation project in particular the CEA-Saclay team, the CERN ATLAS magnet team, JINR workshop Dubna, CERN/AT/ECR cryogenics services and ATLAS BT installation teams.

\section{REFERENCES}

[1] ATLAS Magnet collaboration, The Barrel Toroid Technical Design Report CERN, European Laboratory for Particle Physics, CERN/LHCC 97/10, 1997, ATLAS TDR.

[2] Toroid Assembly_FEA Simulations of the Assembly Phase 3 and Phase 4 internal CEA report, ref 9B $2900 \mathrm{~T}$ - \# 8000003 PA 12/05/2003.

[3] Z. Sun, "ATLAS barrel toroid warm structure design and manufacturing," in Magnet Technology MT19 Conf., Genova, 2005.

[4] — ATLAS TB Warm Structure Calculations on the Bolting Connections reference CERN report EDMS ATL-TB-EA-0005. 